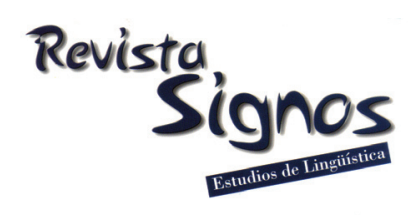

\title{
Aproximación teórico-práctica al procesamiento lingüístico de neologismos léxicos
}

\author{
A theoretical and practical approach to the linguistic \\ processing of neologisms
}

\author{
Carmen Varo \\ Universidad de CÁdiz \\ ESPAÑA \\ carmen.varo@uca.es
}

Recibido: 27-IX-2011 / Aceptado: 16-VIII-2012

\section{Resumen}

El reconocimiento de neologismos puede ser analizado a través de tareas de decisión léxica, como una clase especial de pseudopalabra, cuyos efectos en el proceso de acceso difieren bastante de los constatados en unidades consideradas palabras reales. En el caso de la neología formal, la secuencia fonológica de estas unidades no existe como tal en el lexicón, mientras que, en el caso de la neología semántica, aunque estas unidades estén fonológicamente presentes en el lexicón, estas se vinculan con significados que no se corresponden con el conjunto de contenidos asociados a cada entrada en cuestión. En este sentido, este trabajo se ocupa del procesamiento lingüístico de los neologismos léxicos, atendiendo a cuestiones como el problema de la representación de estas unidades y los modelos elaborados para explicarlo. Asimismo, presentamos un estudio experimental cuyo objeto es determinar los mecanismos cognitivos implicados en el procesamiento de unidades neológicas y las divergencias entre sus modalidades. Este acercamiento práctico nos ha permitido verificar una serie de efectos que contribuyen a la caracterización de este fenómeno desde una perspectiva psicolingüística.

Palabras Clave: Neología, procesamiento lingüístico, neologismo léxico. 


\begin{abstract}
The recognition of neologisms can be analysed through lexical decision tasks that function as a special kind of pseudo-word, whose effects on the accessing process are very different from those shown by units considered real words. In the case of formal neology, the phonological sequence of these units does not exist as such in the lexicon. In the case of semantic neology, although the units are phonologically present in the lexicon, they have meanings that do not appear in the series of content associated to each lexical entry in question. Thus, in this paper we deal with the linguistic processing of lexical neologisms, addressing issues such as the problem of representation of these units and the models created to explain it. Futhermore, we present an experimental study for determining the cognitive mechanisms involved in the processing of neological units and the differences between their various types. This approach allows us to note a series of experimental effects that help characterise this phenomenon from the psycholinguistic perspective.
\end{abstract}

Key Words: Neology, linguistic processing, lexical neologism.

\title{
INTRODUCCIÓN
}

La neología ${ }^{1}$ constituye, sin duda, un gran escollo para el diseño de teorías sobre el procesamiento lingüístico, sobre todo, teniendo en cuenta que la mayoría de estas parten de la aceptación de la existencia de un lexicón mental que explica la rapidez y facilidad para la recuperación léxica. Al no contar con una representación mental previa, el acceso a unidades de nueva creación requiere un modelo alternativo que resuelva el debate sobre la constitución y la organización de nuestro conocimiento léxico. Así, mientras que la neología formal sirve de argumento para la discusión sobre la naturaleza y composición del lexicón, la neología semántica nos lleva a la reflexión sobre la organización semántico-conceptual de nuestro caudal léxico. Por otra parte, como hemos puesto de relieve en trabajos anteriores (Varo, Díaz \& Paredes, 2009), también son notables las limitaciones en los modelos explicativos de la comprensión y la producción léxicas a la hora de dar cuenta de las creaciones neológicas.

En las investigaciones sobre la comprensión léxica, en tanto que la neología de naturaleza semántica se ajusta mejor a los modelos de activación extendida y búsqueda en paralelo (Morton, 1969, 1979), al contemplarse en ellos la participación de estrategias cognitivas que facilitan la asociación semántico-conceptual y la interacción del contexto, precisadas por las metáforas de nueva creación, la neología formal encuentra mejor justificación en los modelos seriales de búsqueda y acceso múltiple (Forster \& Chambers, 1973), por incorporar estos en la memoria léxica representaciones morfológicas ${ }^{2}$, que orientan el proceso de la construcción del significado de la nueva unidad. 
En este trabajo, tras actualizar el estado de la discusión sobre las anteriores cuestiones, abordaremos, a partir de los datos obtenidos mediante la realización de un estudio de carácter experimental, los mecanismos cognitivos esenciales implicados en el procesamiento de unidades neológicas, atendiendo a sus diversos tipos, así como los principales factores que participan en la interpretación de enunciados que incorporan neologismos formales o semánticos, con objeto de:

1) calibrar el grado de eficiencia y la interacción de estos,

2) descubrir singularidades en el procesamiento de unidades nueva creación y

3) sentar las bases para el diseño de un modelo integral de la comprensión neológica.

\section{Posturas sobre el estatus de la neología formal}

En el ámbito de la neología formal ${ }^{3}$ surge el debate sobre el papel de la estructura morfológica en el marco del lexicón mental, pues suele ser asumido por una amplia mayoría de investigadores en Psicolingüística experimental que el acceso léxico, imprescindible tanto para la comprensión como para la producción lingüísticas, solo es posible gracias a un sistema de representación preexistente que opera explícitamente comparando unidades que se han ido almacenando a lo largo de la experiencia vital del individuo, como parte de su memoria. Atendiendo a dichos presupuestos, deberá darse respuesta al problema que ofrecen las formaciones resultantes de una nueva combinación de bases léxicas y afijos, no incluidas, por tanto, en el lexicón de los individuos que, con aparente facilidad, los producen o comprenden por primera vez. En tales circunstancias, podríamos decir de forma intuitiva que, al escuchar o leer una palabra nunca antes procesada, como por ejemplo 'bipremiar' ('premiar dos veces'), probablemente nuestro conocimiento del verbo 'premiar' y del prefijo '-bi' y ciertas normas de configuración morfológica nos orienten en la representación semántica de esa nueva unidad.

En conexión con este tema, es bien conocido el enfrentamiento entre, por un lado, los que piensan las representaciones léxicas aisladas no codifican la estructura morfológica y, por otro, los que consideran que esta información ${ }^{4}$, incorporada al lexicón, es fundamental en los procesos de comprensión y producción léxicas ${ }^{5}$. La primera de ambas posturas, encarnada en la denominada hipótesis de la lista exhaustiva o estrategia basada en la palabra completa (Butterworth, 1983, 1989; Dell \& O’Seaghdha, 1992), es la que peor afronta el inconveniente planteado por la neología formal, puesto que en ella es la palabra la unidad de almacenamiento y acceso.

Por el contrario, el modelo conocido como hipótesis de la lista parcial (Taft \& Forster, 1975), basada en la estrategia del morfema ${ }^{6}$ como unidad de acceso y almacenamiento, en principio parece dar respuesta al problema anterior, pues en el las palabras no se encuentran representadas unitariamente, sino que solo lo están 
sus elementos constituyentes? 7 . De esta forma, para llegar a la representación de formaciones derivadas neológicas como 'archienemigo' o 'ruidista', se tendría que acceder, de un lado, a las raíces 'enemigo' y 'ruido' y, de otro, a los afijos 'archi-' e '-ista', además de conocer las reglas morfológicas para su combinación. Para explicar la forma de acceso a los afijos, no contemplada en los modelos iniciales, Colé, Segui y Taft (1997) propusieron posteriormente que las palabras derivadas y sus raíces compiten en el acceso en función de su frecuencia.

De cualquier forma, desde estos postulados, cada vez que procesamos unidades derivadas se llevaría a cabo una segmentación morfológica, antes de acceder al léxico. Este procedimiento, al margen del incremento en el tiempo de procesamiento y, por ende, del coste cognitivo que conllevaría, no resultaría efectivo en los casos de pseudoafijos. Por ejemplo, en la formación 'bicicletero' ('aficionado a la bicicleta') podemos decir que la segmentación de 'bi-', en lugar de facilitarlo, ralentizará el proceso de acceso léxico. Por último, tampoco se delimitan los efectos morfológicos de los semánticos y de los fónicos, ni se toma en consideración la influencia del contexto en la preactivación de candidatos (Varo et al., 2009).

En las últimas décadas se tiende a abogar por modelos de carácter mixto, como el elaborado por Kiparsky (1982), que justifica las diferencias en el reconocimiento entre unas unidades y otras basándose en diferentes niveles de ligazón de los afijos en función de su productividad, proximidad e interacción con la raíz. En esta línea, se ha sugerido en modelos posteriores que, en tanto que los afijos productivos, fonológicamente neutros y semánticamente transparentes suelen ser descompuestos, aquellos que integran palabras de estructura cambiante y semánticamente opaca no lo son (Marslen-Wilson, Tyler, Waksler \& Older, 1994; Vannest \& Boland, 1999; Waksler, 1999; Wurm, 2000). Por su parte, el modelo de acceso dual ${ }^{9}$ de Burani y Caramazza (1987), si bien incorpora un mecanismo de segmentación morfológica, el acceso léxico no se inicia con el, sino que se apoya en la palabra completa, que a su vez activa la entrada de la raíz, de manera que la activación se propaga a todas las unidades de acceso que comparten el morfema raíz. Desde este marco, el procesamiento de neologismos formales, al no contar estos con una representación léxica previa, solo sería posible a través de unidades léxicas familiares en cuanto a su estructura morfológica y de un proceso de asociación semántica-conceptual.

A la vista de las propuestas analizadas y de las dificultades de aplicación a la neología formal, nos resta hacer una reflexión sobre la naturaleza y composición del lexicón, pues cabe preguntarse si durante el procesamiento el individuo opera partiendo de unidades prealmacenadas ${ }^{10}$ (la diferencia entre los dos modelos consiste en que en los de búsqueda los datos periféricamente guardados son llevados a un manipulador central de símbolos y en los de activación existen distintos manipuladores de símbolos) ya sean léxicas o morfológicas, a las que se accede en función del tipo de palabra, sino más bien un mecanismo de reconstrucción morfológica y léxica, vinculado a los 
sistemas de análisis fonológico secuencial y orientado por factores como la frecuencia y el contexto.

\section{El problema de la representación en los neologismos semánticos}

En la investigación sobre el procesamiento lingüístico son diversas las preocupaciones que surgen respecto a la naturaleza del lexicón mental. De ellas especialmente destacan tres. La primera, de la que nos hemos ocupado en parte en el apartado anterior, es describir cómo se llega a la información léxica a partir de datos sensoriales y contextuales. Las otras dos, en las que nos centraremos en esta ocasión, son explicar la organización de las entradas que constituyen el lexicón y analizar cómo se combinan e integran las diferentes informaciones sémicas almacenadas para lograr construir una representación significativa.

Efectivamente, en lo relativo a la configuración de los conocimientos léxicos, existen opiniones muy dispares sobre si las relaciones semánticas están mentalmente fijadas o no. Si descartamos, como respuesta probable, que estemos programados de forma innata con el conocimiento de todas ellas, dado que cada lengua es la responsable de este, podemos pensar que esta información lingüística es almacenada en la memoria a largo plazo, junto con otros conocimientos lingüísticos, o bien, que dicho conocimiento se genera cada vez que es necesario, a partir de un conjunto de reglas y principios generales (Murphy, 2003). A este respecto, aunque los estudios sobre facilitación semántica (priming), basándose en el tiempo de reconocimiento de palabras precedidas por otras con las que comparten mayor o menor número de rasgos semánticos/conceptuales, apuntan hacia un modelo de organización en redes, también otras pruebas de facilitación confirman que la activación de elementos léxicos asociados igualmente se extiende a unidades de parecido fónico o morfológico, e incluso a unidades cuyos vínculos solo se explican desde la perspectiva experiencial del individuo.

En lo concerniente al reconocimiento de significados de nueva creación y, por tanto, no registrados como parte de la información del lexicón, se ha de tener presente la separación entre metáfora conocida y neología semántica ${ }^{11}$. Así, aunque se ha demostrado mediante estudios experimentales que no hay diferencia en términos de duración temporal o esfuerzo cognitivo en el procesamiento de significados convencionales frente a significados metafóricos (Pollio, Fabrizi, Sills \& Smith, 1984), los resultados son distintos para las metáforas de nueva creación ${ }^{12}$ (Geiger, 1999; Arzouan, Goldstein \& Faust, 2007; Lai, Curran \& Menn, 2009). En cualquier caso, se ha argumentado que las rutas metafóricas ya conocidas sirven para activar nuevas conexiones, sin un gran coste cognitivo (Shibata, Abe, Terao \& Miyamoto, 2007; Thibodeau \& Durgin, 2008). Atendiendo a esta idea, durante el procesamiento de la secuencia 'tan crecido en la indignación como aureolado en la derrota' (véase la Tabla 1), 
recurriríamos a la metáfora convencional 'aureola' = ‘distinción’ para lograr descifrar esta creación semántica. Sin embargo, queda por determinar si necesariamente se ha de acceder primero a los significados completos ya conocidos para poder identificar los nuevos. En esta línea, la lingüística cognitiva explica el fenómeno metafórico como proyección selectiva de las propiedades ${ }^{13}$ de un dominio de partida sobre un dominio meta (Lakoff \& Johnson, 1980; Lakoff, 1993; Rivano, 1997). Asimismo, se ha señalado que durante el procesamiento, mientras que las propiedades asociadas son activadas, otras irrelevantes son inhibidas para que no interfieran en el acceso (McGlone \& Manfredi, 2001).

Otro asunto pendiente de afrontar es la posible interacción de los mecanismos de asociación semántica con los de análisis morfológico, pues ambos deben actuar de manera conjunta en aquellos neologismos en los que además de originarse una nueva estructura formal, el significado interpretado no coincide con la suma de los contenidos de sus constituyentes. Es, por ejemplo, el caso de 'dinerofagia' (véase la Tabla 1), compuesto que trasciende el sentido literal para pasar a interpretarse, en virtud de un proceso metafórico, con el valor de 'codicia'.

\section{Datos a partir de un estudio experimental}

En el marco de la comprensión lingüística el reconocimiento de neologismos puede ser analizado a través de tareas de decisión léxica, al funcionar estos como una clase especial de pseudopalabra, cuyos efectos en el proceso de acceso difieren en gran medida respecto a los manifestados por las unidades consideradas 'palabras legales'.

Con objeto de llevar a cabo una aproximación práctica a los neologismos léxicos desde la perspectiva del procesamiento lingüístico, realizamos un estudio experimental, tomando como referencia dieciséis casos, dentro de sus correspondientes contextos lingüísticos. Dicha muestra trata de recoger distintos procedimientos de formación de palabras, por lo que resulta sumamente útil para descubrir diferencias y similitudes entre ellos. Respecto a los de carácter formal, cuatro se han constituido por prefijación ('archienemigo', 'arracional', 'bipremiar' y 'retradicionalización'), otros cuatro por sufijación ('buenismo', 'ruidista', 'salsarosero' y 'votancia'), uno por parasíntesis ('apalizar') y cuatro por composición culta ('demolingüista', 'dinerofagia', 'discronía' y 'meritocracia'). En cuanto a la neología semántica, incluimos tres casos ('adelgazar', 'aureolar' y 'lavar'), si bien se ha de tener en cuenta que algunos de los neologismos formales utilizados adquieren también un nuevo valor significativo ('ruidista', 'dinerofagia' y 'salsarosero').

Como ya hemos indicado, en los neologismos formales la secuencia fonológica de estas unidades no existe como tal en el lexicón, pese a que, si el sistema lo permite, se puede llevar a cabo su creación (Díaz, 2004), mediante la combinación de otras secuencias existentes en este. En el caso de la neología semántica, aunque fonológicamente nos encontramos con unidades que forman parte del lexicón, estas 
presentan significados que no aparecen consignados en el conjunto de contenidos asociados a cada entrada léxica en cuestión. Todos los ejemplos analizados aparecen recogidos en la Tabla 1, donde se indica también el procedimiento de formación, el significado y el contexto del que proceden.

Tabla 1. Neologismos utilizados en el estudio ( $\mathrm{NS}=$ neologismo semántico, $\mathrm{NF}=$ neologismo formal). Los contextos de uso han sido tomados del banco de neologismos del Centro Virtual Cervantes ().

\begin{tabular}{|c|c|}
\hline $\begin{array}{l}\text { Adelgazar (NS) } \\
\text { 'Reducir en número' }\end{array}$ & $\begin{array}{l}\text { El programa recoge que se intentará *adelgazar* las estructuras } \\
\text { ministeriales }[\ldots] \text {. Fuente: Periódico El País. }\end{array}$ \\
\hline $\begin{array}{l}\text { Apalizar (NF por parasíntesis) } \\
\text { 'Propinar una paliza' }\end{array}$ & $\begin{array}{l}\text { El episodio en el que un labrador *apaliza* a un adolescente está } \\
\text { protagonizado por el nuevo entrenador del Real Madrid [...]. } \\
\text { Fuente: Periódico El País. }\end{array}$ \\
\hline $\begin{array}{l}\text { Archienemigo (NF por prefi- } \\
\text { jación) } \\
\text { 'Enemigo en grado extremo' }\end{array}$ & $\begin{array}{l}\text { Siempre pensaron que el haber colaborado en la persecución de } \\
\text { Pablo Escobar, jefe del cartel de Medellín y su *archienemigo* } \\
\text { muerto en diciembre de 1993, les serviría de garantía. Fuente: } \\
\text { Periódico El País. }\end{array}$ \\
\hline $\begin{array}{l}\text { Arracional (NF por prefijación) } \\
\text { 'Fuera de lo racional' }\end{array}$ & $\begin{array}{l}\text { Se ha llegado a los confines de la razón: la realidad micro y } \\
\text { macrocósmica es irracional o *arracional*. Fuente: Periódico La } \\
\text { Vanguardia. }\end{array}$ \\
\hline $\begin{array}{l}\text { Aureolar (NS) } \\
\text { 'Condecorar' }\end{array}$ & $\begin{array}{l}\text { En cuanto a Mariano Rajoy baste decir por hoy que se le echó de } \\
\text { menos en Canarias junto a su candidato Mayor Oreja, tan crecido } \\
\text { en la indignación como *aureolado* de derrota. Fuente: Periódico } \\
\text { El País. }\end{array}$ \\
\hline $\begin{array}{l}\text { Buenismo (NF por sufijación) } \\
\text { 'Inclinación hacia una actitud } \\
\text { bondadosa' }\end{array}$ & $\begin{array}{l}\text { Edwards es un antiguo abogado y orador brillante -aunque a veces } \\
\text { vacío en su *buenismo* optimista-. Fuente: Periódico El País. }\end{array}$ \\
\hline $\begin{array}{l}\text { Bipremiar (NF por prefijación) } \\
\text { 'Premiar dos veces' }\end{array}$ & $\begin{array}{l}\text { [...] respondón y quisquilloso, recibiendo capones en las madru- } \\
\text { gadas del éter del García-Butanito, ya no queda ni mácula en este } \\
\text { presentador de pista de circo, *bipremiado*, que explica cómo } \\
\text { [...]. Fuente: Periódico La Vanguardia. }\end{array}$ \\
\hline $\begin{array}{l}\text { Demolingüista (NF por com- } \\
\text { posición culta) } \\
\text { 'Lingüista que hace demagogia' }\end{array}$ & $\begin{array}{l}\text { De hecho, las fronteras políticas y las fronteras lingüísticas co- } \\
\text { inciden pocas veces e incluso hay *demolingüistas* que afirman } \\
\text { que no existe ningún Estado totalmente monolingüe. Fuente: } \\
\text { Periódico El País. }\end{array}$ \\
\hline $\begin{array}{l}\text { Dinerofagia (NF por composición } \\
\text { culta). } \\
\text { 'Ansia de acumular dinero' }\end{array}$ & $\begin{array}{l}\text { El cuerpo de la Comunidad Valenciana, víctima de la *dinerofa- } \\
\text { gia* de políticos y especuladores, ha empezado ya a dar síntomas } \\
\text { preocupantes. Fuente: Periódico El País. }\end{array}$ \\
\hline $\begin{array}{l}\text { Discronía (NF por composición } \\
\text { culta) } \\
\text { 'Ausencia o problema de coinci- } \\
\text { dencia' }\end{array}$ & $\begin{array}{l}\text { Esta *discronía* política parece ponernos en situación de apren- } \\
\text { der de los errores ajenos. Fuente: Periódico La Vanguardia. }\end{array}$ \\
\hline $\begin{array}{l}\text { Lavar (NS) } \\
\text { 'Enmascarar’ }\end{array}$ & $\begin{array}{l}{[\ldots] \text { después de que el Sodbusinessbank fuera privado en mayo de }} \\
\text { su licencia, acusado de *lavar* dinero y financiar el terrorismo. } \\
\text { Fuente: Periódico El País. }\end{array}$ \\
\hline $\begin{array}{l}\text { Meritocracia (NF por com- } \\
\text { posición culta) } \\
\text { 'Gobierno o poder obtenido por } \\
\text { méritos' }\end{array}$ & $\begin{array}{l}\text { O a la falta de } * \text { meritocracia* ya que no se paga más a quien mejor } \\
\text { trabaja y no se promociona a quien se lo merece, sino a quien } \\
\text { mejores conexiones tiene. Fuente: Periódico La Vanguardia. }\end{array}$ \\
\hline
\end{tabular}




\begin{tabular}{|l|l|}
\hline $\begin{array}{l}\text { Retradicionalización (NF por } \\
\text { prefijación) } \\
\text { 'Proceso para volver a convertir } \\
\text { algo en tradición' }\end{array}$ & $\begin{array}{l}\text { Las identidades son también mercancía, y todos los procesos de } \\
\text { redefinición y *retradicionalización*-tan frecuentes hoy- se reali- } \\
\text { zan bajo el dominio del imperativo económico. Fuente: Periódico } \\
\text { El País. }\end{array}$ \\
\hline $\begin{array}{l}\text { Ruidista (NF por sufijación). } \\
\text { 'Destinado a hacer ruido o hac- } \\
\text { erse notar' }\end{array}$ & $\begin{array}{l}\text { Los neoyorquinos continúan subidos a su atalaya *ruidista* y no } \\
\text { es cuestión de cambiar a estas alturas. Fuente: Periódico El País. }\end{array}$ \\
\hline $\begin{array}{l}\text { Salsarosero (NF por sufijación) } \\
\text { 'Aficionado a los programas de } \\
\text { cotilleo' }\end{array}$ & $\begin{array}{l}\text { La carencia [...] de los recursos activistas desarrollados en las } \\
\text { tradiciones feministas, maricas, bolleras, transexuales, transgénero } \\
\text { e intersexuales en el estado español se ve compensada hoy por un } \\
\text { *alsarosero* y vitaminado saber mediático. Fuente: Periódico La } \\
\text { Vanguardia. }\end{array}$ \\
\hline $\begin{array}{l}\text { Votancia (NF por sufijación). } \\
\text { 'Colectivo que puede votar' }\end{array}$ & $\begin{array}{l}\text { Pero sobre lo que vaya a hacer el domingo la *votancia*, ni idea. } \\
\text { Fuente: Periódico El País. }\end{array}$ \\
\hline
\end{tabular}

Los enunciados, ordenados aleatoriamente, en los que aparecen estos neologismos fueron presentados de forma escrita a un total de cincuenta informantes pertenecientes a un grupo homogéneo de individuos (estudiantes del primer curso de licenciatura, cuya lengua materna era el español), que respondieron de manera individual a cinco cuestiones:

¿Conocía o utilizó antes la palabra destacada?

¿Qué piensa que significa? ¿Se le ocurren sinónimos?

¿Qué proceso ha seguido para llegar al significado?

¿Ha tenido que leerla más de una vez o invertir más tiempo para comprender su significado?

¿Conocería su significado fuera de contexto?

Las respuestas a la primera pregunta nos sirvieron para detectar casos en los que tales unidades léxicas ya eran conocidas por los informantes y, como consecuencia, debían ser descartados. Las preguntas segunda y tercera nos permitieron comprobar si el nuevo significado era procesado de forma correcta, teniendo en cuenta la intención comunicativa de la fuente de la que se extraen. Las preguntas fundamentales del estudio son las dos últimas, en las que se pretende conocer el esfuerzo cognitivo invertido y las estrategias seguidas en el proceso interpretativo.

Los datos obtenidos aparecen reflejados en las tablas que aparecen abajo. La Tabla 2 se basa en el número de encuestados que necesitaron leer más de una vez los enunciados que incluían neologismos. La Tabla 3 se relaciona con el número de encuestados que utilizaron la información contextual para reconstruir el sentido del enunciado. 
Tabla 2. Porcentajes que reflejan de menor a mayor el incremento en el tiempo de procesamiento.

\begin{tabular}{|l|l|}
\hline Archienemigo & $10 \%$ \\
\hline Lavar & $25 \%$ \\
\hline Apalizar & $27 \%$ \\
\hline Adelgazar & $30 \%$ \\
\hline Bipremiar & $35 \%$ \\
\hline Arracional & $40 \%$ \\
\hline Ruidista & $45 \%$ \\
\hline Salsarosero & $50 \%$ \\
\hline Meritocracia & $55 \%$ \\
\hline Votancia & $60 \%$ \\
\hline Dinerofagia & $68 \%$ \\
\hline Retradicionalización & $70 \%$ \\
\hline Demolingüista & $72 \%$ \\
\hline Aureolar & $72 \%$ \\
\hline Buenismo & $80 \%$ \\
\hline Discronía & $90 \%$ \\
\hline
\end{tabular}

Tabla 3. Porcentajes que reflejan de menor a mayor la utilización del contexto.

\begin{tabular}{|l|l|}
\hline Apalizar & $6 \%$ \\
\hline Archienemigo & $6 \%$ \\
\hline Adelgazar & $6 \%$ \\
\hline Bipremiar & $6 \%$ \\
\hline Retradicionalización & $30 \%$ \\
\hline Aureolar & $30 \%$ \\
\hline Lavar & $35 \%$ \\
\hline Arracional & $40 \%$ \\
\hline Ruidista & $45 \%$ \\
\hline Salsarosero & $50 \%$ \\
\hline Meritocracia & $55 \%$ \\
\hline Votancia & $64 \%$ \\
\hline Buenismo & $70 \%$ \\
\hline Dinerofagia & $70 \%$ \\
\hline Demolingüista & $80 \%$ \\
\hline Discronía & $90 \%$ \\
\hline
\end{tabular}


Como se aprecia en la Tabla 1, atendiendo al tipo de formación, en cuanto al tiempo de reconocimiento, sobre todo algunos de los compuestos cultos considerados precisaron con una frecuencia bastante elevada una nueva lectura del enunciado en el que se incluían ('dinerofagia' $=68 \%$, 'demolingüista' $=72 \%$ y 'discronía' $=90 \%$ ), aunque también otras clases de formaciones arrojan porcentajes que oscilan entre el $70 \%$ y el $90 \%$ ('retradicionalización' $=70 \%$, 'aureolar' $=72 \%$ y 'buenismo' $=80 \%$ ), lo que nos permite concluir que la modalidad neológica no constituye un factor único para explicar la diferencias registradas. No obstante, los compuestos cultos fueron las formaciones que requirieron con mayor frecuencia la apelación al contexto para su interpretación, como se muestra en la Tabla 2 ('dinerofagia' $=70 \%$, 'demolingüista' $=$ $80 \%$ y 'discronía' $=90 \%$ ). Tales observaciones pueden explicarse por la menor frecuencia de algunos de los elementos integrantes de estas formaciones, factor que adquiere mayor relevancia que la naturaleza formal o semántica de las unidades neológicas. Este hecho se refuerza, teniendo en cuenta que, ni desde el punto de vista del tiempo de procesamiento ni atendiendo a la participación del contexto se observan diferencias destacables entre neologismos por derivación y neologismos semánticos.

Parece que la relectura y la información contextual se utilizan en mayor o menor medida dependiendo de la eficiencia de los mecanismos de análisis de la estructura formal y del significado de las unidades implicadas, como se desprende de los resultados singularizados correspondientes a las modalidades de neología usadas en el estudio, que presentamos a continuación.

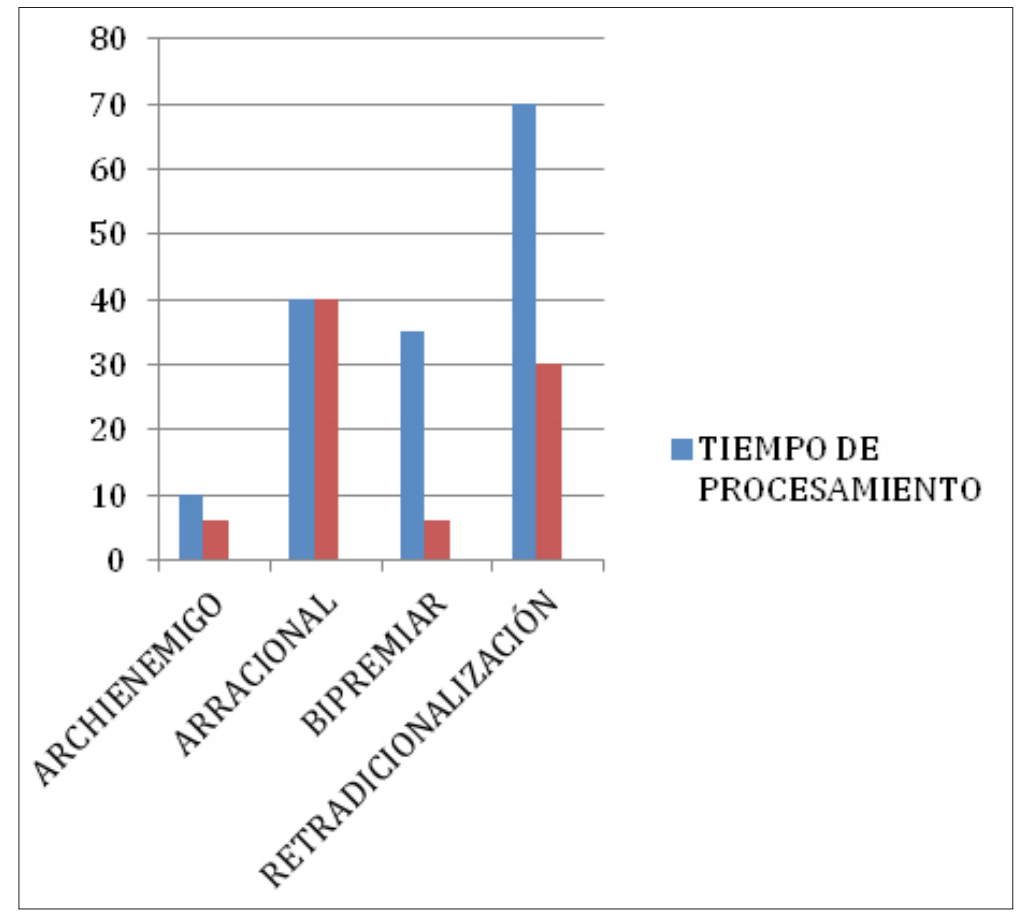

Gráfico 1. Porcentajes para neologismos formales por prefijación. 
La dispersión de los valores obtenidos para las formaciones por prefijación puede relacionarse con la transparencia semántica de los prefijos y su posible confusión con secuencias silábicas de palabras no derivadas. Así, frente al valor inequívoco del prefijo 'archi-', 're-' muestra mayor tendencia a la ambigüedad.

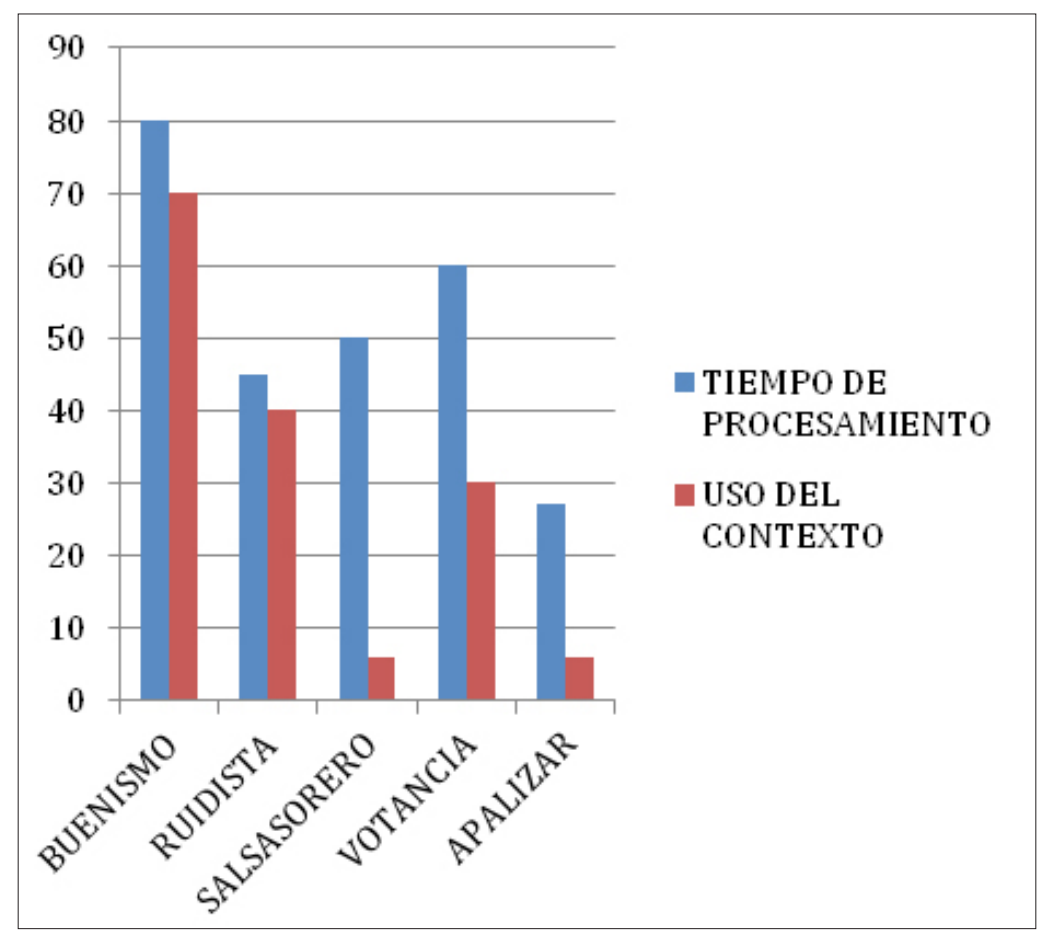

Gráfico 2. Porcentajes para neologismos formales por sufijación y parasíntesis.

Las formaciones derivadas por sufijación, con porcentajes superiores a los derivados por prefijación en cuanto a la dificultad de procesamiento, presentan menor dispersión de valores y las diferencias entre los neologismos estudiados pueden justificarse por la frecuencia de los sufijos, mayor en '-ista' y '-ero'. Respecto de la formación por parasíntesis, la suma de procedimientos formales para la creación léxica no parece aumentar el tiempo de procesamiento ni, por consiguiente, el esfuerzo cognitivo, en relación con los otros tipos. No obstante, este dato, por basarse en un único caso, necesita ser comprobado en mayor número de ejemplos. 


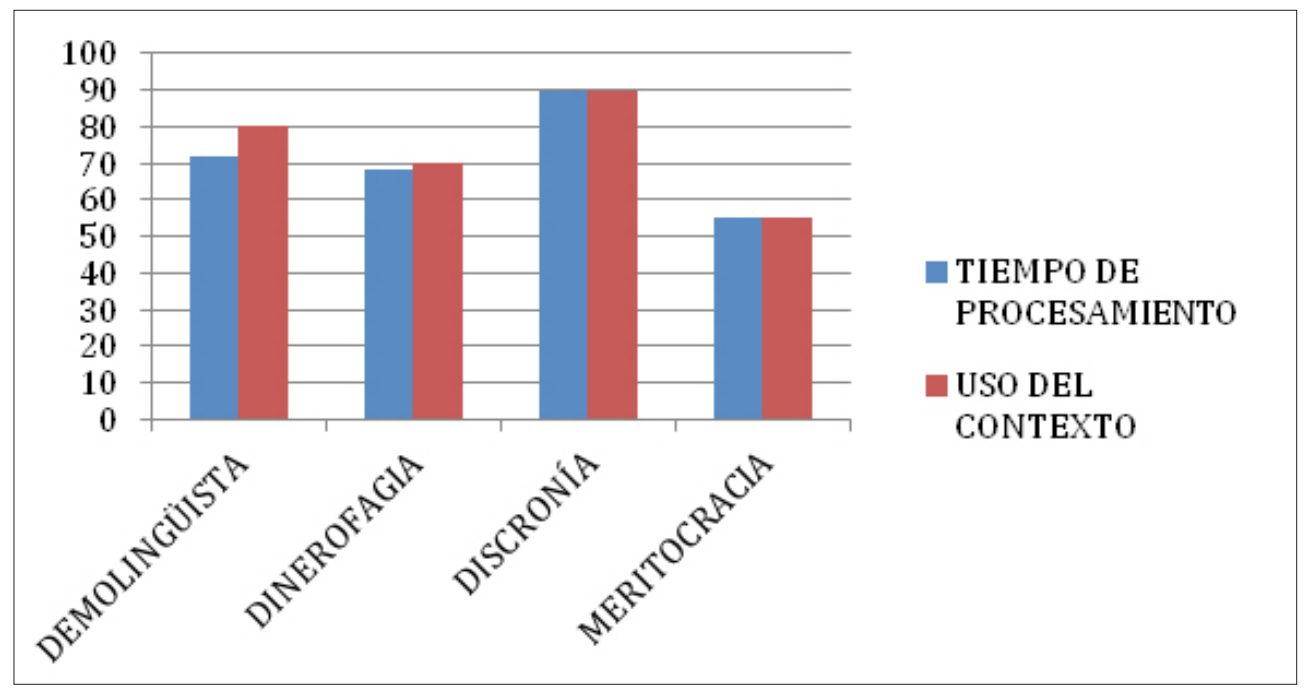

Gráfico 3. Porcentajes para neologismos formales por composición.

La menor eficiencia de los mecanismos de reconstrucción formal, sumada al bajo índice de frecuencia de algunos de los constituyentes de los compuestos analizados, podría ser la explicación del aumento en el uso de la información contextual en este tipo de neologismos, en comparación con los anteriores. Respecto a las diferencias entre los casos considerados, el procesamiento de las formaciones 'dinerofagia' y 'meritocracia' frente a los otros compuestos, probablemente se ve favorecido por la familiaridad de los encuestados con las unidades 'dinero' y 'mérito'.

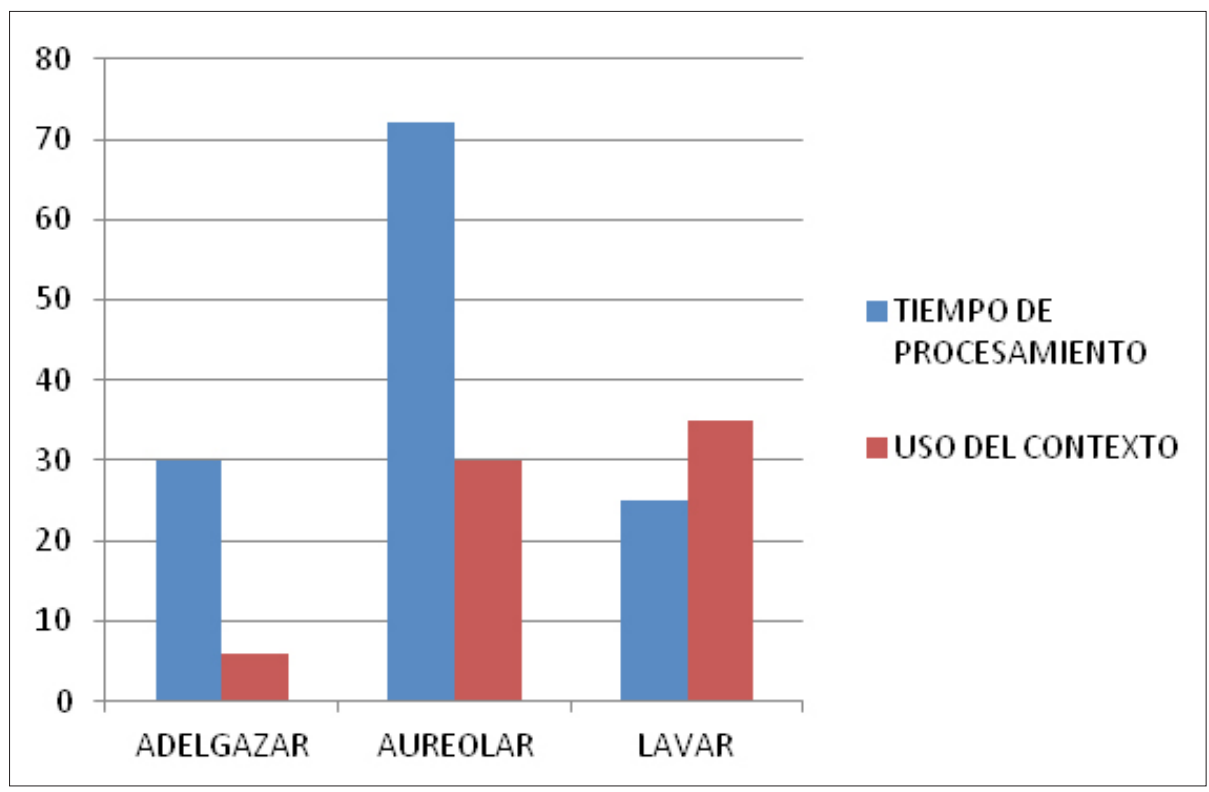

Gráfico 4. Porcentajes para neologismos semánticos. 
De nuevo la frecuencia (menor de 'aureolar') vuelve a ser la única posible explicación de las diferencias entre los tres casos estudiados. La recurrencia al contexto es llamativamente alta en el caso de 'lavar', pese a que solo un $25 \%$ de los encuestados invirtió más tiempo en su reconocimiento. En este comportamiento puede influir la distancia semántica del valor neológico 'enmascarar' frente a los rasgos habituales manifestados por esta unidad.

\section{Discusión de resultados: Mecanismos cognitivos implicados en el procesamiento neológico}

La aproximación a la neología a través de tareas de decisión léxica para recabar información sobre la forma de procesamiento y el esfuerzo cognitivo invertido por los encuestados, contribuye a la caracterización de este fenómeno desde una perspectiva psicolingüística. Tras el estudio realizado, queremos resaltar algunos mecanismos mentales y efectos experimentales que nos permiten avanzar en algunas de las propuestas teóricas presentadas en investigaciones anteriores y configuran un modelo plausible de procesamiento neológico.

En primer lugar, sin valorar algunos casos en que ya se conocían los neologismos, todos los encuestados lograron interpretar adecuadamente los enunciados en que aparecían insertos. Este hecho se puede explicar por el enorme poder apelativo de las tales creaciones, que conduce al individuo a la búsqueda de rutas de acceso que pueden diferir de las establecidas para el procesamiento de unidades ya conocidas. En este marco adquiere especial protagonismo la habilidad cognitiva de la atención o prominencia, relacionada con los grados de activación de las estructuras conceptuales, en la que participan procesos como la selección o habilidad para atender a partes de nuestra experiencia relevantes para un determinado propósito o ignorar aspectos irrelevantes para tal propósito (Croft \& Cruse, 2004).

Durante la reconstrucción del sentido de los neologismos, queda de manifiesto en la capacidad de los encuestados la actuación de un mecanismo de análisis morfológico, en el que la detección de determinados patrones del sistema lingüístico en cuestión es fundamental, pues este facilita especialmente el procesamiento de nuevas creaciones léxicas de carácter formal, aunque para ello sea necesario invertir mayor esfuerzo cognitivo, sobre todo en algunos casos.

Las unidades léxicas creadas mediante prefijación (por ejemplo, 'archienemigo'= $10 \%$ y 'bipremiar' $=35 \%$ ), frente a las formadas por sufijación (por ejemplo, 'buenismo' $=80 \%$ y 'votancia' $=60 \%$ ), parecen mostrar, en líneas generales, mayor facilidad para ser procesadas, lo que confirma la linealidad del proceso de comprensión y la relevancia de la porción inicial de la palabra como clave para el acceso.

En conexión con el analizador morfológico, otro mecanismo esencial que hemos observado en la interpretación de neologismos es la búsqueda de asociaciones conceptuales. El acierto en el procesamiento de las creaciones semánticas utilizadas 
en nuestro estudio (pese a que en un porcentaje superior al $25 \%$ fue necesaria la lectura de los enunciados que los incluían), nos hacen pensar que estas no son interpretadas a través del significado convencional completo, sino que son solo ciertos rasgos semánticos los que facilitan la reconstrucción del significado, con lo que se reduce considerablemente el esfuerzo cognitivo y, como consecuencia, el tiempo invertido. De ahí que, por ejemplo, no sea imprescindible acceder al contenido canónico completo de 'adelgazar' en el enunciado 1, sino simplemente a alguno de las marcas que lo configuran ('reducir'), favorecido por un marco cognitivo (programa de actuación política) que nada tiene que ver con cuestiones de salud o mantenimiento físico y que, por tanto, inhibe otras marcas semánticas a las que en otros contextos también se suele propagar la activación. Por otra parte, es evidente que existe interacción entre los mecanismos de análisis de la estructura formal y la configuración semántica, pues no se incrementa la dificultad de comprensión en las formaciones en las que, además de un proceso derivativo, se da un proceso metafórico, como, por ejemplo, en 'ruidista' y 'salsarosero', que incluso se interpretan con mayor facilidad que otros neologismos formales en los que no hay proceso metafórico.

Los datos obtenidos en nuestro experimento, principalmente, apuntan hacia un efecto experimental: la frecuencia, factor que ha sido ampliamente tratado en la bibliografía sobre procesamiento léxico (Bradley \& Forster, 1987). Ahora bien, en los neologismos actúa de una forma particularmente distinta, ya que en lugar de considerarse atendiendo a la palabra completa se basa en los constituyentes de esta. Así, por ejemplo, el reconocimiento de creaciones neológicas como 'bipremiar' y 'ruidista' dependerá en gran medida de la frecuencia con la que el interpretante procesa unidades como 'premiar' y 'ruido' y afijos como 'bi-' e '-ista'. Sin duda, la participación de un mecanismo de reconstrucción léxica basado en la frecuencia justifica el hecho de que los neologismos por composición culta, utilizados en nuestro estudio, muestren datos estadísticos que reflejan un mayor tiempo de procesamiento, así como la recurrencia al contexto para su interpretación.

De manera secundaria, en la comprensión de neologismos formales y semánticos son destacables los efectos contextuales, también comúnmente señalados en la bibliografía sobre procesamiento léxico. Respecto al primer tipo, se ha comprobado en estudios anteriores que responden a las pruebas de facilitación por repetición en función del mecanismo morfológico de creación léxica utilizado. A través de diversas pruebas experimentales, Colé, Beauvillain y Segui (1989) demostraron que los tiempos de decisión léxica son menores para palabras sufijadas precedidas de otras palabras que contenían los mismos sufijos. En cambio, observaron que el acceso a palabras prefijadas no es facilitado por las propiedades morfológicas comunes de las palabras precedentes. Esto se confirma en casos como 'retradicionalización' (el $70 \%$ de los encuestados necesitaron volver a leer el enunciado), cuyo procesamiento es más lento, en relación con otras formaciones neológicas, incluso cuando aparece precedida, como en el enunciado utilizado en nuestra prueba, de otro sustantivo 
con el que comparte prefijo ('redefinición'). Ahora bien, también hemos constatado que la presencia en el mismo enunciado de unidades que comparten la base léxica, como en el adjetivo 'arracional', precedido de 'irracional', tampoco facilitó mucho el procesamiento (el 40\% de los encuestados leyeron de nuevo el enunciado). A ello contribuye la polivalencia del prefijo 'a-', que obliga a activar distintas posibilidades significativas.

Igualmente, podemos explicar algunos de los datos recabados como resultado de ciertos efectos derivados de la facilitación contextual. En ese sentido, se explica el procesamiento de casos como 'salsarosero', precedido en el ejemplo de un claro marco explicativo ('La carencia [...] de los recursos activistas desarrollados en las tradiciones feministas, maricas, bolleras, transexuales, transgénero e intersexuales [...]').

Otro interesante efecto que se observa en la neología formal, el de la superioridad de la palabra, en el sentido de que el tiempo empleado en identificar una palabra completa es inferior al tiempo invertido en identificar sus componentes (Burani \& Caramazza, 1987), se puede constatar en el procesamiento de 'votancia' o 'buenismo', cuyos elevados porcentajes en los dos indicadores utilizados parecen indicar que el análisis de los componentes constituye un mecanismo postléxico. En el caso de 'buenismo', se añade un curioso efecto relacionado con el parecido entre esta palabra y el superlativo 'buenísimo', que parece ralentizar el procesamiento.

Por último, debemos añadir otros efectos experimentales semánticos como la imaginabilidad, o facilidad para evocar o imaginar el significado léxico, y la capacidad significativa, o número de significados asociados a una palabra, constatable en el procesamiento de 'adelgazar', cuyo rasgo semántico principal 'reducir' es aplicable en múltiples situaciones. Además, hemos de mencionar, respecto a la comprensión de ambos tipos de neologismos, la importancia de la disponibilidad contextual o facilidad que un sujeto tiene para imaginar un contexto o circunstancia concretos en los que pudiera ocurrir la palabra que se pretende reconocer. Por ejemplo, la estructura 'acusado de lavar dinero' (11), inserta en un texto en el que se explica un delito desde el punto de vista del hablante facilitará la interpretación de la unidad 'lavar', no solo por el contexto semántico, sino también por el sintáctico.

\section{CONCLUSIONES}

El estudio del procesamiento lingüístico de los neologismos constituye uno de los pilares esenciales para la caracterización de la neología, así como para la delimitación particular de cado uno de sus diferentes modalidades, atendiendo a los mecanismos mentales que sustentan dichos procesos y en los que es preciso seguir ahondando, con la ampliación del corpus de ejemplos y de las pruebas empleadas. Asimismo, este tipo de análisis debe ser complementado por la indagación de las bases neurológicas de la neología, mediante técnicas de neuroimagen como la tomografía por emisión de positrones (PET) o la resonancia magnética funcional (fMRI). 
Como hemos tratado de mostrar, más allá de la necesidad puramente denominativa, no siempre justificada, por existir otras posibilidades léxicas ya instaladas en la norma de la comunidad hablante, el recurso a la neología, desde una vertiente psicolingǘstica, se conecta con una estrategia comunicativa de marcada tendencia apelativa, dirigida a captar con extraordinaria eficacia la atención de destinatario del mensaje, al precisar la interpretación de este la participación de mecanismos mentales (y, por ende, de estructuras cerebrales) diferenciados de los normalmente empleados en el procesamiento del léxico ya conocido.

Según se ha visto en los dos primeros apartados, en el marco de la neología formal, el debate se ha centrado en determinar qué clase de representaciones, morfológicas o léxicas, maneja el individuo durante el procesamiento, en tanto que en las consideraciones sobre la neología semántica se tiende a dar por sentado que se accede a las nuevas interpretaciones a través de significados canónicos.

Frente a estos planteamientos, teniendo en cuenta los resultados obtenidos tras nuestra investigación, nos parece más oportuno abordar la descripción del procesamiento neológico de acuerdo con el enfoque componencial presente en el paradigma estructuralista, como un proceso de reconstrucción de las unidades lingüísticas, tanto desde el punto de vista de la estructura formal como de su configuración semántica, orientado por patrones de frecuencia y facilitado por la información sensorial y conceptual proporcionada en el contexto en el que se incluyen. En este sentido, nos inclinamos por un modelo que integre de manera simultánea un sistema de reconstrucción morfológica y léxica y otro de análisis semánticoconceptual, que puedan interactuar durante el procesamiento, dirigidos por el mecanismo cognitivo de la atención y afectados por múltiples efectos relacionados con la experiencia lingüística acumulada por el interpretante. 


\section{REFERENCIAS BIBLIOGRÁFICAS}

Ahrens, K., Liu, H., Lee, C., Gong, S., Fang, S. \& Hsu, S. (2007). Functional MRI of conventional and anomalous metaphors in Mandarin Chinese. Brain and Language, 100, 163-171.

Arzouan, Y., Goldstein, A. \& Faust, M. (2007). Dynamics of hemispheric activity during metaphor comprehension: Electrophysiological measures. Neuroimage, 36(1), 222-231.

Bottini, G., Corcoran, R., Sterzi, R., Paulesu, E., Schenone, P., Scarpa, P., Frackowiak, R. S. \& Frith, D. (1994). The role of the right hemisphere in the interpretation of figurative aspects of language: A positron emission tomography activation study. Brain, 117, 1241-1253.

Bradley, D. C. \& Forster, K. I. (1987). A reader's view of listening. Cognition, 25, 103134.

Burani, C. \& Caramazza, A. (1987). Representation and processing of derived words. Language and Cognitive Processes, 2, 217-227.

Butterworth, B. (1983). Lexical representation. En B. Butterworth (Ed.), Language production (pp. 257-294). Londres: Academic Press.

Butterworth, B. (1989). Lexical access in speech production. En W. Marslen-Wilson (Ed.), Lexical representation and process (pp. 108-135). Cambridge, MA: MIT Press.

Colé, P., Beauvillain, C. \& Segui, J. (1989). On the representation and processing of prefixed and suffixed derived words. A differential frequency effect. Journal of Memory and Language, 28, 1-13.

Colé, P., Segui, J. \& Taft, M. (1997). Words and morphemes as units for lexical access. Journal of Memory and Language, 37, 312-330.

Croft, W. \& Cruse, D. A. (2004). Cognitive linguistics. Cambridge: Cambrigde University Press.

Dell, G. \& O’ Seaghdha, P. (1992). Stages of lexical access in language production. Cognition, 42, 287-314.

Díaz, M. T. (2004). Restricciones del sistema y restricciones de la norma en la formación de palabras. Lingüistica en la Red II [en línea]. Disponible en: http://www2.uah.es/ linred, http://www.linred.com

Díaz, M. T. (2008). La investigación lingüística de la neología léxica en España. Estado de la cuestión. LynX. Panorámica de Estudios Lingüísticos, 7, 5-60. 
Feldman, L. B. (1994). Beyond orthography and phonology: Differences between inflections and derivations. Journal of Memory and Language, 33, 442-470.

Forster, K. I. \& Chambers, S. M. (1973). Lexical access and naming time. Journal of Verbal Learning and Verbal Behaviour, 12, 627-635.

Frauenfelder, U. \& Schreuder, R. (1992). Constraining psycholinguistic models of morphological processing and representation: The role of productivity. En G. Booij \& J. van Marle (Eds.), Yearbook of morphology (pp. 165-183). Dordrecht: Kluwer.

Geiger, O. (1999). Metaphors and the mental lexicon. Brain and Language, 68, 190-198.

Glucksberg, S. (2003). The psycholinguistics of metaphor. Trends in Cognitive Science, 7, 92-96.

Kiparsky, P. (1982). Lexical phonology and morphology. En I. Yang (Ed.), Linguistics in the morning calm (pp. 3-91). Seúl: Hanshin.

Lai, V. T., Curran, T. \& Menn, L. (2009). Comprehending conventional and novel metaphors: An ERP study. Brain Research, 1284, 145-155.

Lakoff, G. (1993). The contemporary theory of metaphor. En A. Ortony (Ed.), Metaphor and thought (pp. 202-251). Cambridge: Cambridge University Press.

Lakoff, G. \& Johnson, M. (1980). Metaphors we live by. Chicago: University of Chicago Press.

Lamb, S. (1999). Pathways of the brain. The neurocognitive basis of language. Amsterdam/ Philadelphia: John Benjamins.

Marangolo, P., Incoccia, C., Pizzamiglio, L., Sabatini, U., Castriota-Scanderbeg, A. \& Buran, C. (2003). The right hemisphere involvement in morphologically derived words. Journal of Cognitive Neuroscience, 15, 364-371.

Marslen-Wilson, W., Tyler, L., Waksler, R. \& Older, L. (1994). Morphology and meaning in the English mental lexicon. Psychological Review, 101(1), 3-33.

Mashal, N., Faust, M., Hendler, T. \& Jung-Beeman, M. (2007). An fMRI investigation of the neural correlates underlying processing of novel metaphoric expressions. Brain and Language, 100, 115-126.

McGlone, M. \& Manfredi, D. (2001). Topic-vehicle interaction in metaphor comprehension. Memory and Cognition, 29, 1209-1219.

McQueen, J. \& Cutler, A. (1998). Morphology in word recognition. En A. Zwicky \& A. Spencer (Ed.), The handbook of morphology (pp. 406-427). Oxford: Blackwell. 
Miceli, G. \& Caramazza, A. (1988). Dissociation of inflectional and derivational morphology. Brain and Language, 35, 24-65.

Morton, J. (1969). Interaction of information in word recognition. Psychological Review, 76, 165-178.

Morton, J. (1979). Facilitation in word recognition: Experiments causing change in the logogen model. En P. A. Kolers, M. E. Wrolstad \& H. Bouma (Eds.), Processing of visible language (pp. 259-268). Nueva York: Plenum.

Murphy, L. M. (2003). Semantic relations and the lexicon. Cambridge: Cambridge University Press.

Pinker, S. (1991). Rules of language. Science, 253, 530-535.

Pollio, H., Fabrizi, M., Sills, A. \& Smith, M. (1984). Need metaphoric comprehension take longer than literal comprehension? Journal of Psycholinguistic Research, 13(3), 195-214.

Pynte, J., Besson, M., Robichon, F. \& Poli, J. (1996). The time-course of metaphor comprehension: An event-related potential study. Brain and Language, 55, 293316.

Rivano, E. (1997). Metáfora y lingüística cognitiva. Santiago de Chile: Bravo y Allende Editores.

Schmidt, G., DeBuse, C. \& Seger, C. (2005). Right hemisphere metaphor processing. Characterizing the lateralization of semantic processes. Brain and Language, 100, 127-141.

Shibata, M., Abe, J., Terao, A. \& Miyamoto, T. (2007). Neural mechanims involved in the comprehension of metaphoric and literal sentences: An fMRI study. Brain Research, 1166, 92-102.

Taft, M. (1994). Interactive-activation as a framework for understanding morphological processing. Language and Cognitive Processes, 9, 271-294.

Taft, M. \& Forster, K. (1975). Lexical storage and retrieval of prefixed words. Journal of Verbal Learning and Verbal Behavior, 14, 638-647.

Thibodeau, P. \& Durgin, F. H. (2008). Productive figurative communication: Conventional metaphors facilitate the comprehension of related novel metaphors. Journal of Memory and Language, 58, 521-540.

Tyler L. \& Marslen-Wilson, W. (1997). Disorders of combination: Processing of complex words. Brain and Language, 60, 40-48. 
Waksler, R. (1999). Cross-linguistic evidence for morphological representation in the mental lexicon. Brain and Language, 68, 68-74.

Vannest, J. \& Boland, J. (1999). Lexical morphology and lexical access. Brain and Language, 68, 324-332.

Varo, C., Díaz, M. T. \& Paredes, M. J. (2009). Modelos comunicativos y producción e interpretación neológicas. Revista de Investigación Lingüistica, 12, 185-216.

Wurm, L. H. (2000). Auditory processing of polymorphemic pseudowords. Journal of Memory and Language, 42(2), 255-271.

\section{NOTAS}

1. Una revisión de las definiciones de los términos 'neología' y 'neologismo' en diccionarios de lengua, diccionarios de lingüística, obras lexicográficas de terminología lingüística y estudios monográficos de los más representativos investigadores del fenómeno figura en Díaz (2008).

2. Ambos tipos de modelos entienden el análisis morfológico como uno de los mecanismos implicados en la comprensión del lenguaje. La diferencia entre ellos radica en el orden y la forma de intervención de este, dado que en los de acceso directo se contempla una preactivación de diversas posibilidades, gracias a la participación, desde el principio, de múltiples analizadores lingüísticos, que interactúan con otros mecanismos cognitivos y con la información del exterior, en tanto que para los de búsqueda la selección se realiza en una fase de postacceso, mediante un proceso de verificación entre distintas opciones a partir de la información previa del contexto.

3. Aunque los procedimientos formales de creación neológica son numerosos, nos centraremos en esta ocasión, por motivos de extensión, en los basados en la derivación y la composición.

4. En las primeras fases de la investigación sobre procesamiento morfológico, Taft y Forster (1975) proponían que las palabras prefijadas son analizadas en sus morfemas constituyentes antes de que tenga lugar el acceso léxico. Esta concepción inicial con el tiempo evoluciona hacia un modelo de doble ruta (Taft, 1994).

5. Un resumen de las posturas surgidas en torno a este tema puede verse en McQueen y Cutler (1998).

6. No obstante, atendiendo a esta propuesta, la palabra perdería protagonismo como base para el reconocimiento léxico, pues serían los morfemas las unidades en las que se apoya el procesamiento, al contar estos con su propia representación de entrada (Varo et al., 2009).

7. La relevancia del análisis morfológico ha sido constatada por investigaciones neurolingüísticas sobre el asiento biológico de este mecanismo, de acuerdo con sus modalidades flexiva y derivativa (Burani \& Caramazza, 1987; Miceli \& Caramazza, 1988; Feldman, 1994; Tyler \& Marslen-Wilson, 1997; Marangolo, Incoccia, Pizzamiglio, Sabatini, Castriota-Scanderbeg \& Buran, 2003).

8. La mayoría de los ejemplos de neologismos utilizados en este estudio (ver Tabla 1), aparecidos en El País y La Vanguardia, están tomados del banco de neologismos del Centro Virtual Cervantes: http://cvc.cervantes.es/obref/banco_neologismos/

9. Otros modelos de alistamiento dual (Pinker, 1991; Frauenfelder \& Schreuder, 1992; Feldman, 1994) también postulan un componente léxico en el cual están representadas las relaciones morfológicas, posiblemente de manera independiente de las relaciones semánticas y sintácticas, de tal forma que los efectos morfológicos deberían ser observados en el mismo componente en el que se encuentran las entradas léxicas.

10. La hipótesis del almacenamiento de información simbólica, compartida por gran número de investigadores de distintas tendencias, ha sido especialmente criticada desde el marco de lingüística neurocognitiva por Lamb (1999: 375), que en relación con esta cuestión señala: "The mind is not a device for storing and rewriting symbols but a network system, whose information is in its conectivity". 
11. Frente a una mayoría de trabajos sobre la comparación neurológica del procesamiento del significado literal frente al de metáforas convencionales, son relativamente escasos los dedicados a las bases biológicas de las metáforas de nueva creación (Bottini, Corcoran, Sterzi, Paulesu, Schenone, Scarpa, Frackowiak \& Frith 1994; Pynte, Besson, Robichon \& Poli, 1996; Geiger, 1999; Schmidt, DeBuse \& Seger, 2005; Ahrens, Liu, Lee, Gong, Fang \& Hsu, 2007; Arzouan et al., 2007; Mashal, Faust, Hendler \& Jung-Beeman, 2007; Lai et al., 2009).

12. Desde el punto de vista de sus bases biológicas, el procesamiento de neologismos semánticos puede ser singularizado, frente al procesamiento de unidades léxicas de significado literal e incluso de metáforas convencionales, por la actuación de mecanismos propios de un análisis holista, característico del hemisferio derecho, imprescindible para la producción y la comprensión de nuevos significados léxicos. La participación del hemisferio derecho en ciertos tipos de creación léxica, a partir del estudio de pacientes afásicos, es estudiada en Marangolo et al. (2003).

13. Para explicar la conexión entre la acepción de lengua y la metafórica, han surgido modelos como el de inclusión de clases de Glucksberg (2003), en el que se contempla cierta vinculación polisémica entre ambas. Esta propuesta se ajusta mejor a la metáfora convencional que a las creaciones neológicas de naturaleza semántica, pues el vínculo entre los dos significados implicados es muchas veces bastante débil. 